\title{
Wavelet-Based Homotopy Analysis Method for Nonlinear Matrix System and Its Application in Burgers Equation
}

\author{
Xing Ruyi \\ Hebei University of Engineering, Handan 056038, China \\ Correspondence should be addressed to Xing Ruyi; xingruyi_hue@163.com \\ Received 17 March 2013; Accepted 14 June 2013 \\ Academic Editor: Evangelos J. Sapountzakis \\ Copyright (C) 2013 Xing Ruyi. This is an open access article distributed under the Creative Commons Attribution License, which \\ permits unrestricted use, distribution, and reproduction in any medium, provided the original work is properly cited. \\ To generalize the homotopy analysis method (HAM) to multidegree-of-freedom nonlinear system, the adaptive precise integration \\ method (APIM) is introduced into the HAM, with which the almost exact value of the exponential matrix can be obtained. \\ Combining the interval interpolation wavelet collocation method, HAM-based APIM can be employed to solve the nonlinear PDEs. \\ As an example, Burgers equation is spatially discretized by the interval quasi-Shannon wavelet collocation method and solved by \\ the proposed method to illustrate the effectiveness and great potential of the homotopy analysis method in nonlinear problems.
}

\section{Introduction}

The homotopy analysis method (HAM) is developed in 1992 by Liao in [1-5]. This method has been successfully applied in various nonlinear problems in science and engineering in [611] and references therein. Based on homotopy of topology, the validity of the HAM is independent of whether or not there exist small parameters in the considered equation. Therefore, the HAM can overcome the foregoing restrictions and limitations of perturbation techniques [12,13]. In fact, $\mathrm{He}$ $[14,15]$ proposed another asymptotic numerical method for solving nonlinear problems named homotopy perturbation method (HPM). The better improvement is adding an auxiliary parameter into the homotopy equation, which is helpful to eliminate the secular term in the perturbation solution. This can improve the rate of convergence greatly. Unlike analytical perturbation methods, HPM does not depend on small parameter which is difficult to find. Most researchers think of HPM as a special case of HAM, which is much simpler than classical HAM and has been widely used in solving various nonlinear problems [16]. However, the HAM applies mainly in single-degree-of-freedom systems; as for multidegree-offreedom nonlinear differential equations, due to the correlation of the equations, the solving process is very tedious, and strong calculating technique is needed.
In an attempt to overcome the previous difficulties, the precise integration method (PIM) proposed by Zhong [17] in 1994 is employed, because it is effective in computation precision and stability for the solutions of linear differential equations $[18,19]$. However, like other numerical methods, PIM has its own limitation. When it is applied to the problems of time-variant or nonlinear time integration, PIM usually expands the nonlinear term in Taylor series firstly, which is then solved by using an iteration method. In order to obtain high precision, a very small time step should be taken for PIM, which results in huge computational complexity and greatly restricts the application of PIM in engineering.

Burgers' model of turbulence is a crucial fluid dynamic model, and the study of this model and theory of shock waves has been considered by many authors both for conceptual understanding of a class of physical flows and for testing various numerical methods. In this work, the Burgers equation is firstly transformed into a set of nonlinear matrix differential equations using wavelet collocation method [20-22]. Since the definition domain of wavelet transformation is an infinite interval, which can deduce the boundary effect, this can be eliminated by introducing an interval wavelet [23] instead of the common wavelet function.

Taking full advantage of the HAM and PIM, a novel approximate method for multidegree-of-freedom nonlinear 
matrix differential equation based on interval wavelet is presented in this paper.

\section{The HAM Based on the PIM}

2.1. Basic Idea of the Homotopy Analysis Method. Taking as an example, we consider the following nonlinear algebraic equation:

$$
f(x)=0
$$

First of all, we construct such a homotopy

$$
H(x ; q)=(1-q)\left[f(x)-f\left(x_{0}\right)\right]+q \cdot f(x),
$$

where $x_{0}$ is an initial guess of $x$ and $q \in[0,1]$ is called embedding parameter. Obviously, at $q=0$ and $q=1$, one has

$$
H(x ; 0)=f(x)-f\left(x_{0}\right), \quad H(x ; 1)=f(x),
$$

respectively. Thus, as $q$ increases from 0 to $1, H(x ; q)$ varies continuously from $f(x)-f\left(x_{0}\right)$ to $f(x)$. Such kind of continuous variation is called deformation in topology [20]. Now, enforcing $H(x ; q)=0$, that is,

$$
(1-q)\left[f(x)-f\left(x_{0}\right)\right]+q \cdot f(x)=0,
$$

we have now a family of algebraic equations. Obviously, the solution of the previous family of algebraic equations is dependent upon the embedding parameter $q$. So, the family of equations can be rewritten as

$$
(1-q)\left\{f[\phi(x ; q)]-f\left(x_{0}\right)\right\}+q \cdot f[\phi(x ; q)]=0 .
$$

As the embedding parameter $q$ increases from 0 to 1 , $\phi(x ; q)$ varies from the initial guess $x_{0}$ to the solution $x$ of $f(x)=0$. We call the family of equations like (5) the zerothorder deformation equation

Because $\phi(x ; q)$ is now a function of the embedding parameter $q$, we can expand it into Maclaurin series

$$
\phi(x ; q)=x_{0}+\sum_{m=1}^{+\infty} x_{m} \cdot q^{m}
$$

where $\phi(x ; 0)=x_{0}$ is employed, and

$$
x_{m}=\left.\frac{1}{m !} \cdot \frac{\partial^{m} \phi(x ; q)}{\partial q^{m}}\right|_{q=0} .
$$

Here, the series (6) is called homotopy series, and $x_{m}$ is called the $k$ th-order homotopy derivative of $\phi(x ; q)$. If the convergence radius of the previous Maclaurin series is no less than 1 , using the relationship $\phi(x ; 1)=x$, one has

$$
x=x_{0}+\sum_{m=1}^{+\infty} x_{m}
$$

According to the fundamental theorem of calculus about Taylor series, the coefficient $x_{m}$ of the homotopy series (6) is unique.
Substituting (6) into (5) and rearranging based on powers of $q$-terms, one has

$$
\begin{gathered}
x_{1} f^{\prime}\left(x_{0}\right)+f\left(x_{0}\right)=0, \\
x_{2} f^{\prime}\left(x_{0}\right)+\frac{1}{2} x_{1}^{2} f^{\prime \prime}\left(x_{0}\right)=0 .
\end{gathered}
$$

Whose solutions are

$$
\begin{gathered}
x_{1}=-\frac{f\left(x_{0}\right)}{f^{\prime}\left(x_{0}\right)}, \\
x_{2}=-\frac{x_{1}^{2} f^{\prime \prime}\left(x_{0}\right)}{2 f^{\prime}\left(x_{0}\right)}=-\frac{f^{2}\left(x_{0}\right) f^{\prime \prime}\left(x_{0}\right)}{2\left[f^{\prime}\left(x_{0}\right)\right]^{3}} .
\end{gathered}
$$

In this way, one obtains $x_{m}$ one by one in the order $m=$ $1,2,3, \ldots$ Then one has 1st-order homotopy-series approximation

$$
x \approx x_{0}+x_{1}=x_{0}-\frac{f\left(x_{0}\right)}{f^{\prime}\left(x_{0}\right)}
$$

2nd-order homotopy-series approximation

$$
x \approx x_{0}+x_{1}+x_{2}=x_{0}-\frac{f\left(x_{0}\right)}{f^{\prime}\left(x_{0}\right)}-\frac{f^{2}\left(x_{0}\right) f^{\prime \prime}\left(x_{0}\right)}{2\left[f^{\prime}\left(x_{0}\right)\right]^{3}} .
$$

2.2. The Precise Integration Method for Nonlinear Matrix Differential Equations. Taking as an example, we consider the following matrix differential equations:

$$
\frac{d v}{d t}=H v+f(v, t)
$$

where $v$ is an $n$-dimensional unknown vector, $H$ and $f(v, t)$ are $n \times n$ matrix, and $t$ is a time variable. The general solution of (14) is

$$
v(t)=e^{H\left(t-t_{k}\right)} v\left(t_{k}\right)+e^{H t} \int_{t_{k}}^{t} e^{-H t} \cdot f(v, t) d t .
$$

To solve the integral part of (15), the linear interpolation approximation is employed

$$
f(v, t)=r_{0}+r_{1}\left(t-t_{k}\right)
$$

where $r_{0}=f\left(v\left(t_{k}\right), t_{k}\right), r_{1}=r_{1}=\left.(\partial f / \partial t)\right|_{t=t_{k}}+$ $\sum_{i=1}^{2 n}\left[\left(\partial f / \partial v_{i}\right)\left(H v\left(t_{k}\right)+f\left(v\left(t_{k}\right), t_{k}\right)\right)_{i}\right]$, and $v_{i}$ is the $i$ th element of vector $v$. Based on the previous derivations, the recurrence formula of (14) can be obtained as

$$
\begin{aligned}
v\left(t_{k+1}\right)= & e^{H\left(t_{k+1}-t_{k}\right)}\left[v\left(t_{k}\right)+H^{-1}\left(r_{0}+H^{-1} r_{1}\right)\right] \\
& -H^{-1}\left[r_{0}+H^{-1} r_{1}+\left(t-t_{k}\right) r_{1}\right] .
\end{aligned}
$$

Supposing the time step $\tau=t_{k+1}-t_{k}$ is constant, the matrix exponential function can be calculated accurately by using PIM. The calculation of the exponential function is based on the identity

$$
e^{H \cdot \tau}=\left[e^{H \cdot(\tau / m)}\right]^{m}
$$

where $m$ is an integer. It is suggested to select $m=2^{N}$, such as $N=20, m=1048576$. 
Because $\tau$ is a small time interval, $\Delta t=\tau / m$ is an extremely small time interval. Hence for the interval, the truncated Taylor expansion is applied with high precision

$$
T=e^{H \cdot \Delta t}=I+T a,
$$

where $T a=H \cdot \Delta t+(H \cdot \Delta t)^{2} / 2+(H \cdot \Delta t)^{3} / 3 !+(H \cdot \Delta t)^{4} / 4 !$

Because $\Delta t$ is very small, the first five-term series expansion should be enough.

For computing the matrix $T$, (17) could be factored as

$$
T=[I+T a]^{2^{N}}=[I+2 \cdot T a+T a \cdot T a]^{2^{N-1}} .
$$

Such factorization should be iterated $N$ times. Noted that it is equivalent to do the following instruction:

$$
\begin{gathered}
\text { for }(\text { iter }=0 ; \text { iter! }=N ;++ \text { iter }) \\
T a=2 \cdot T a+T a \cdot T a,
\end{gathered}
$$

after the execution of the above instruction, $T=I+T a$.

Because the nonlinear term $f(v, t)$ is expanded in Taylor series and the PIM uses an iterative method, the time step $\tau$ must be very small.

\section{Coupling Technique of the HAM and the PIM for Burgers Equation Based on Interval Wavelet}

3.1. Spatial Discretization of the Burgers Equation. Consider that the one-dimensional Burgers equation has the form [2123]

$$
\frac{\partial u}{\partial t}+u \frac{\partial u}{\partial x}=\frac{1}{\operatorname{Re}} \frac{\partial^{2} u}{\partial^{2} x}, \quad x \in[0,2], t \geq 0
$$

subject to the initial and boundary conditions

$$
u(x, 0)=\sin (\pi x), \quad u(0, t)=u(2, t)=0,
$$

where $t$ represents the time, while Re denotes the Reynolds number.

According to the basic idea of wavelet collocation method, the solution $u(x, t)$ could be approximately represented as

$$
u(x, t) \approx u_{j}(x, t)=\sum_{n=0}^{2^{j}} u\left(x_{n}, t\right) \varphi_{j}\left(x-x_{n}\right)
$$

where $x_{n}\left(n=0,1,2, \ldots, 2^{j}\right)$ are uniform discrete points and $\varphi(x)$ is the scaling function. Here we take the quasi-Shannon wavelet $[24,25]$ as the scaling function

$$
\varphi(x)=\frac{\sin (\pi x)}{\pi x} \exp \left(-\frac{x^{2}}{2 \sigma^{2}}\right), \quad \sigma>0,
$$

where $\sigma$ is the window size parameter.
In order to take the quasi-Shannon scaling function as the basis function, $u(x, t)$ is discretized in its definition domain

$$
x_{n}=2^{1-j} \cdot n, \quad j \in Z, n=0,1,2, \ldots, 2^{j} .
$$

And thereby the basis function is obtained

$$
\varphi_{j}\left(x-x_{n}\right)=\frac{\sin \left[2^{j-1} \pi\left(x-x_{n}\right)\right]}{2^{j-1} \pi\left(x-x_{n}\right)} \exp \left[-\frac{2^{2 j-3}\left(x-x_{n}\right)^{2}}{r^{2}}\right],
$$

where $r=2^{j-1} \cdot \sigma$.

It is easy to see that $\varphi_{j}\left(x-x_{n}\right)$ has the interpolation property

$$
\varphi_{j}\left(x_{k}-x_{n}\right)=\delta_{k, n}= \begin{cases}1 & k=n \\ 0 & k \neq n\end{cases}
$$

Using its interpolation property, the first derivative and second derivative of $\varphi_{j}\left(x-x_{n}\right)$ can be obtained as follows:

$$
\begin{aligned}
& \varphi_{j}^{\prime}\left(x-x_{n}\right) \\
& = \begin{cases}0 & k=n \\
\frac{2^{j-1} \cos [\pi(k-n)] \exp \left[-(k-n)^{2} / 2 r^{2}\right]}{(k-n)} & k \neq n,\end{cases} \\
& \varphi_{j}^{\prime \prime}\left(x-x_{n}\right) \\
& = \begin{cases}-\frac{\left(3+\pi^{2} r^{2}\right) \cdot 2^{2 j-2}}{3 r^{2}} & k=n \\
-2^{2 j-2} \cdot\left[\frac{1}{(k-n)^{2}}+\frac{1}{r^{2}}\right] \cdot \cos [\pi(k-n)] & \\
\cdot \exp \left[-\frac{(k-n)^{2}}{2 r^{2}}\right] & k \neq n .\end{cases}
\end{aligned}
$$

Substituting (4) into (1), a series of nonlinear ODEs can be obtained

$$
\begin{aligned}
& \frac{d u\left(x_{k}, t\right)}{d t} \\
& \quad=\sum_{n=0}^{2^{j}} u\left(x_{n}, t\right)\left[\frac{1}{\operatorname{Re}} \varphi_{j}^{\prime \prime}\left(x_{k}-x_{n}\right)-u\left(x_{k}, t\right) \varphi_{j}^{\prime}\left(x_{k}-x_{n}\right)\right] .
\end{aligned}
$$

For simplicity, we can write (10) as matrix differential equation

$$
\frac{d V(t)}{d t}=M_{0} \cdot V(t)+M_{1}(V(t)) \cdot V(t),
$$


where

$$
\begin{gathered}
V(t)=\left[u\left(x_{0}, t\right), u\left(x_{1}, t\right), \ldots, u\left(x_{2^{j}}, t\right)\right]^{T}, \\
M_{0}=\frac{1}{\operatorname{Re}}\left(\begin{array}{cccc}
\varphi_{j}^{\prime \prime}\left(x_{0}-x_{0}\right) & \varphi_{j}^{\prime \prime}\left(x_{0}-x_{1}\right) & \cdots & \varphi_{j}^{\prime \prime}\left(x_{0}-x_{2^{j}}\right) \\
\varphi_{j}^{\prime \prime}\left(x_{1}-x_{0}\right) & \varphi_{j}^{\prime \prime}\left(x_{1}-x_{1}\right) & \cdots & \varphi_{j}^{\prime \prime}\left(x_{1}-x_{2^{j}}\right) \\
\vdots & \vdots & \cdots & \vdots \\
\varphi_{j}^{\prime \prime}\left(x_{2^{j}}-x_{0}\right) & \varphi_{j}^{\prime \prime}\left(x_{2^{j}}-x_{1}\right) & \cdots & \varphi_{j}^{\prime \prime}\left(x_{2^{j}}-x_{2^{j}}\right)
\end{array}\right), \\
M_{1}=-\operatorname{diag}(V(t)) \\
\cdot\left(\begin{array}{ccc}
\varphi_{j}^{\prime}\left(x_{0}-x_{0}\right) & \varphi_{j}^{\prime}\left(x_{0}-x_{1}\right) \cdots & \varphi_{j}^{\prime}\left(x_{0}-x_{2^{j}}\right) \\
\varphi_{j}^{\prime}\left(x_{1}-x_{0}\right) & \varphi_{j}^{\prime}\left(x_{1}-x_{1}\right) \cdots & \varphi_{j}^{\prime}\left(x_{1}-x_{2^{j}}\right) \\
\vdots & \vdots & \vdots \\
\varphi_{j}^{\prime}\left(x_{2^{j}}-x_{0}\right) & \varphi_{j}^{\prime}\left(x_{2^{j}}-x_{1}\right) \cdots & \varphi_{j}^{\prime}\left(x_{2^{j}}-x_{2^{j}}\right)
\end{array}\right) .
\end{gathered}
$$

3.2. Construction of Quasi-Shannon Interval Wavelet. The Lagrange polynomials $l_{j, k}^{1}, l_{j, k}^{2}$ are introduced

$$
l_{j, k}^{1}=\prod_{\substack{i=0 \\ i \neq k}}^{L} \frac{x-x_{j, i}}{x_{j, k}-x_{j, i}}, \quad l_{j, k}^{2}=\prod_{\substack{i=2^{j}-L \\ i \neq k}}^{2^{j}} \frac{x-x_{j, i}}{x_{j, k}-x_{j, i}} .
$$

The following two weight coefficients are defined

$$
a_{n k}=l_{j, k}^{1}\left(x_{j n}\right), \quad b_{n k}=l_{j, k}^{2}\left(x_{j n}\right) .
$$

Then the interpolation basic function can be expressed as

$$
\begin{gathered}
w_{j, k}(x)=w_{j, k}(x)+\sum_{n=-N+1}^{-1} a_{n k} \cdot w_{j, n}(x), \quad k=0, \ldots, L, \\
w_{j, k}(x)=w_{j, k}(x), \quad k=L+1, \ldots, 2^{j}-L-1, \\
w_{j, k}(x)=w_{j, k}(x)+\sum_{n=2^{j}+1}^{2^{j}+N-1} b_{n k} \cdot w_{j, n}(x), \\
k=2^{j}-L, \ldots, 2^{j},
\end{gathered}
$$

where $L$ is the number of he external points and $N$ is the support domain of the wavelet function.

It is easy to know that the quasi-Shannon interval wavelet is a linear combination of the quasi-Shannon scaling function $w_{j, k}(x)$. Therefore, the quasi-Shannon interval wavelet function possesses all the properties of the quasi-Shannon scaling function.

3.3. The PIM for Matrix Differential Equations. We choose the time step as $\tau$, assuming that the nonlinear term in (31) is linear in the relatively short time segment $\left(t_{k}, t_{k+1}\right)$, the nonlinear term $M_{1}(V(t)) \cdot V(t)$ of (31) can be expressed in Taylor series, and the first two terms are taken. Therefore, (31) could be represented as

$$
\frac{d V(t)}{d t}=M_{0} V(t)+r_{0}+r_{1}\left(t-t_{k}\right)
$$

where

$$
\begin{gathered}
r_{0}=M_{1}\left(t_{k}\right) V\left(t_{k}\right), \\
r_{1}=\left[M_{2}\left(t_{k}\right)+M_{1}\left(t_{k}\right) M_{0}+M_{1}\left(t_{k}\right) M_{1}\left(t_{k}\right)\right] V\left(t_{k}\right), \\
M_{2}(t)=\frac{d M_{1}(t)}{d t} .
\end{gathered}
$$

Based on the previous derivation, the iterative solution of (38) can be obtained

$$
\begin{aligned}
V\left(t_{k+1}\right)= & e^{M_{0} \cdot \tau}\left[V\left(t_{k}\right)+M_{0}^{-1}\left(r_{0}+M_{0}^{-1} r_{1}\right)\right] \\
& -M_{0}^{-1}\left(r_{0}+M_{0}^{-1} r+r_{1} \tau\right),
\end{aligned}
$$

where the matrix exponential function $e^{M_{0} \cdot \tau}$ can be calculated accurately by using PIM.

3.4. The Homotopy Analysis Method Based on the Precise Integration Method. There are various ways to construct a homotopy function. For (11), a linear homotopy can be constructed as

$$
\begin{aligned}
& q\left[\frac{d \phi(x, t ; q)}{d t}-M_{0}-M_{1}(\phi(x, t ; q)) \phi(x, t ; q)\right] \\
& +(1-q) \\
& \quad \times\left[\frac{d \phi(x, t ; q)}{d t}-M_{0} \phi(x, t ; q)-M_{1}(A) \phi(x, t ; q)\right] \\
& \quad=0,
\end{aligned}
$$

where $A=V(0)$ is the initial approximation of $V(t)$ and $q \epsilon$ $[0,1]$ is the embedding parameter. As $q$ increases from 0 to 1 , the solution $\phi(x, t ; q)$ varies from the initial guess $A$ to the solution $V(t)$, and it can be expanded in Taylor series about the embedding parameter

$$
\phi(x, t ; q)=A+\sum_{m=1}^{+\infty} V_{m}(t) \cdot q^{m}
$$

where

$$
V_{m}(t)=\frac{1}{m !} \frac{\partial^{m} \phi(x, t ; q)}{\partial q^{m}}
$$

that the approximate solutions are generally not valid for large $t$. In order to improve the computation accuracy, we divide the time interval $[0-t]$ into a number of sections uniformly by setting the time step as $\tau$, and use the iterative method. In such a way, a series of time nodes can be expressed as

$$
t_{0}=0, \quad t_{1}=\tau, \ldots, \quad t_{k}=k \cdot \tau, \ldots
$$

Since the only requirement for $q$ is that from $q=0$ to $q=$ $1, \phi(x, t ; q)$ should change continuously from the initial guess 
$A$ to $V(t)$. Therefore, it is feasible to improve the computation accuracy by assuming that

$$
q=\frac{\Delta t}{\tau}, \quad \Delta t \in[0, \tau] .
$$

Then $\phi(x, t ; q)$ can be expanded in the form

$$
\begin{aligned}
\phi(x, t ; q)= & V_{0}(t)+V_{1}(t) \cdot\left(\frac{\Delta t}{\tau}\right)+V_{2}(t) \cdot\left(\frac{\Delta t}{\tau}\right)^{2} \\
& +V_{3}(t) \cdot\left(\frac{\Delta t}{\tau}\right)^{3}+\cdots .
\end{aligned}
$$

Substituting (46) into (41) and rearranging based on powers of $q$-terms, the following are obtained:

$$
\begin{gathered}
V_{0}(t)=A, \\
\frac{d V_{0}(t)}{d t}=H \cdot V_{0}(t)=H \cdot A, \\
V_{1}(t)=\tau \cdot \frac{d V_{0}(t)}{d t}=\tau \cdot H \cdot A, \\
\frac{d V_{1}(t)}{d t}=H \cdot V_{1}(t)_{0}=\tau \cdot H^{2} \cdot A, \\
\frac{d V_{2}(t)}{d t}=H \cdot V_{2}(t)+s_{0}=\frac{\tau^{2} H^{3}}{2} \cdot A+s_{0}, \\
V_{3}(t)=\frac{\tau}{3} \cdot \frac{d V_{2}(t)}{d t}=\frac{(\tau H)^{3}}{3 !} \cdot A+\frac{\tau}{3} \cdot s_{0}, \\
\frac{d V_{3}(t)}{d t}=H \cdot V_{3}(t)+s_{1}=\frac{\tau^{3} H^{4}}{3 !} \cdot A+\frac{\tau H}{3} \cdot s_{0}+s_{1}, \\
V_{4}(t)=\frac{\tau}{4} \cdot \frac{d V_{4}(t)}{d t}=\frac{(\tau H)^{4}}{4 !} \cdot A+\frac{\tau^{2} H}{3 \cdot 4} \cdot s_{0}+\frac{\tau}{4} \cdot s_{1},
\end{gathered}
$$

where $s_{0}=M_{1}\left(V_{1}(t)\right) \cdot A, s_{1}=M_{1}\left(V_{1}(t)\right) \cdot V_{1}(t)+M_{1}\left(V_{2}(t)\right)$. $V_{0}(t)$ and $H=M_{0}+M_{1}(A)$.

Substituting (47) into (46) and assuming $q=1$, one has

$$
\begin{aligned}
V(t)= & V_{0}(t)+V_{1}(t)+V_{2}(t)+V_{3}(t)+\cdots \\
= & {\left[I+\tau H+\frac{(\tau H)^{2}}{2 !}+\cdots\right] A } \\
& +\left[\frac{\tau}{3}+\frac{\tau^{2} H}{3 \cdot 4}+\frac{\tau^{3} H^{2}}{3 \cdot 4 \cdot 5}+\cdots\right] s_{0} \\
& +\left[\frac{\tau}{4}+\frac{\tau^{2} H}{4 \cdot 5}+\frac{\tau^{3} H^{2}}{4 \cdot 5 \cdot 6}+\cdots\right] s_{1}+\cdots \\
= & T \cdot A+2 \cdot \frac{\left(H^{-1}\right)^{3}}{\tau^{2}}\left[\frac{(\tau H)^{3}}{3 !}+\frac{(\tau H)^{4}}{4 !}+\cdots\right] s_{0} \\
& +3 ! \cdot \frac{\left(H^{-1}\right)^{4}}{\tau^{3}}\left[\frac{(\tau H)^{4}}{4 !}+\frac{(\tau H)^{5}}{5 !}+\cdots\right] s_{1}+\cdots \\
= & T \cdot A+2 \cdot \frac{\left(H^{-1}\right)^{3}}{\tau^{2}} \cdot R \cdot s_{0} \\
& +3 ! \cdot \frac{\left(H^{-1}\right)^{4}}{\tau^{3}} \cdot Q \cdot s_{1}+\cdots,
\end{aligned}
$$

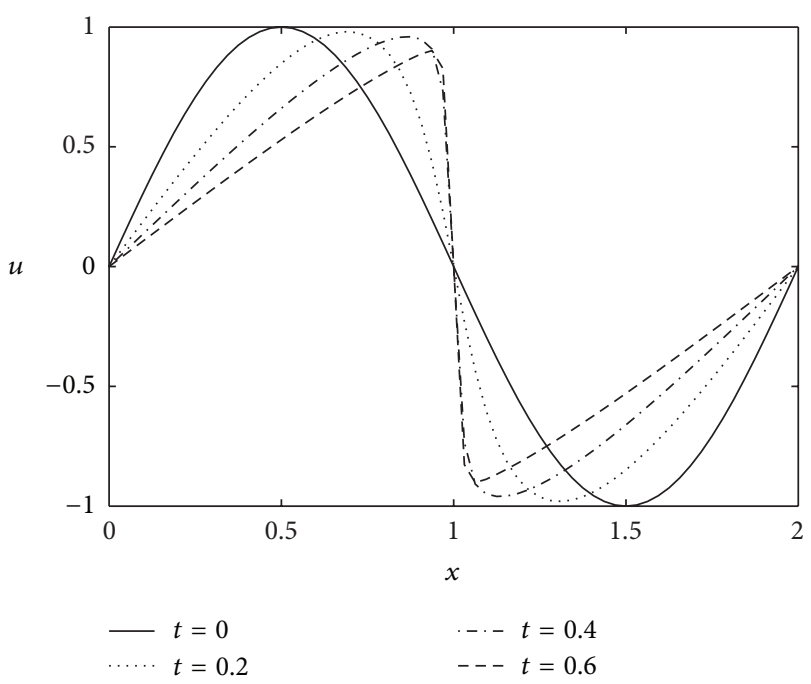

Figure 1: Analytical solutions of the Burgers equation at different times $(t=0,0.4,0.6)$.

where $R=\left[T-I-\tau H-(\tau H)^{2} / 2\right], Q=R-(\tau H)^{3} / 6, T=e^{\tau H}$. The matrix exponential function $T$ can be calculated by using PIM.

For simplicity, only the first two terms are taken as the approximate solution. Accordingly, the approximate solution is obtained

$$
V(t) \approx V_{0}(t)+V_{1}(t)=T \cdot A+2 \cdot \frac{\left(H^{-1}\right)^{3}}{\tau^{2}} \cdot R \cdot s_{0} .
$$

\section{Numerical Results and Discussion}

The Burgers equation has analytical solution as follows:

$$
\begin{aligned}
& u(x, t) \\
& =\frac{4 \pi}{\operatorname{Re}} \cdot \sum_{n=1}^{\infty} n \exp \left(-\frac{n^{2} \pi^{2} t}{\operatorname{Re}}\right) I_{n}\left(\frac{\mathrm{Re}}{2 \pi}\right) \sin (n \pi x) \\
& \quad \times\left(2 \sum_{n=1}^{\infty} n \exp \left(-\frac{n^{2} \pi^{2} t}{\operatorname{Re}}\right) I_{n}\left(\frac{\mathrm{Re}}{2 \pi}\right) \cos (n \pi x)+I_{0}\left(\frac{\mathrm{Re}}{2 \pi}\right)\right)^{-1},
\end{aligned}
$$

where $I_{n}(x)$ is the $n$th order modified Bessel function of the first kind. Figure 1 shows the analytical solutions with $\mathrm{Re}=$ 100 at $t=0,0.4,0.6$, respectively. It is easy to see that the analytical solution evolves into a shock wave near $x=1$, and the gradient becomes larger and larger with the increasing of Re.

In the analysis of the numerical result, we choose Reynolds number $\operatorname{Re}=100$, the scaling parameter $j=8$, the artificial parameter $r=3$, and the number of the external points $L=4$.

The comparison of the classical PIM and the HAM is shown in Figures 2 and 3 and Tables 1 and 2.

We can see clearly that when the time step $\tau$ is relatively large ( $\tau=0.05$ or $\tau=0.1$ ), the classical PIM fails actually it fails when $\tau$ is 0.04 . However, the HAM has enough precision even when the time step $\tau$ increases to 0.1 and $t$ is more than 0.6 . 


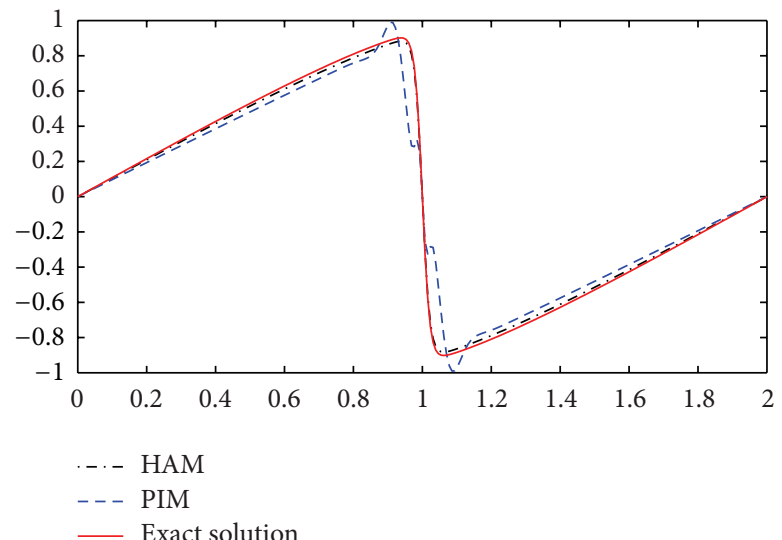

(a) $\tau=0.05, t=0.6$

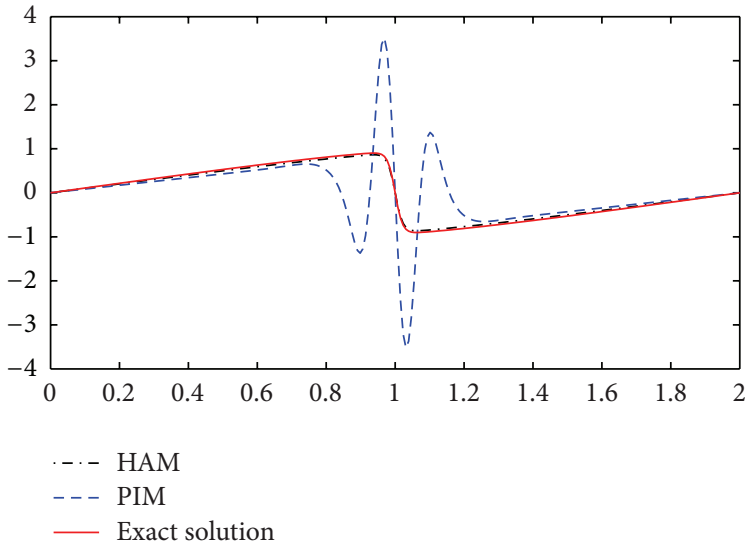

(b) $\tau=0.1, t=0.6$

FIGURE 2: Numerical results of the classical PIM and the HAM.

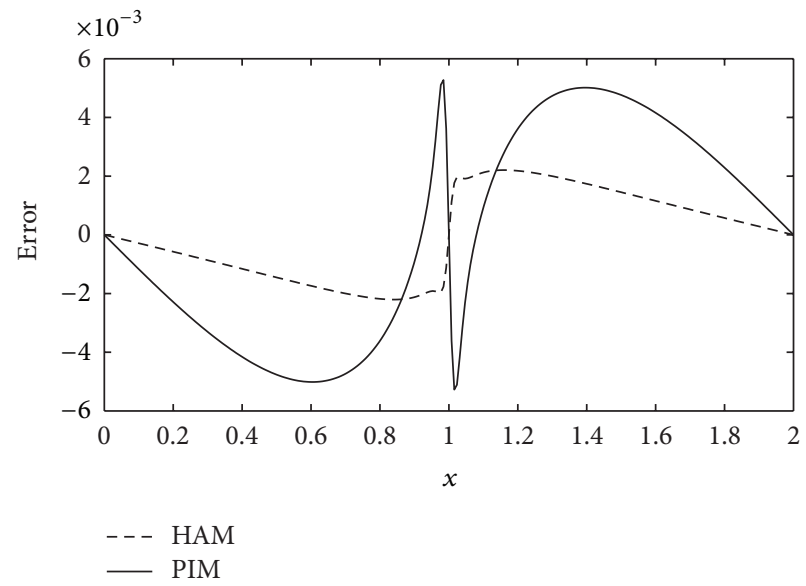

(a) $\tau=0.005, t=0.6$

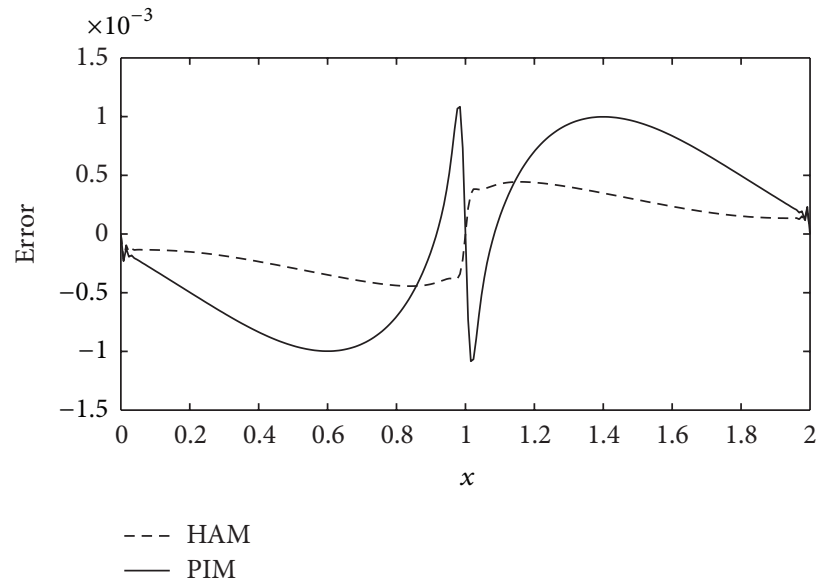

(b) $\tau=0.001, t=0.6$

FIGURE 3: Errors of the classical PIM and the HAM.

TABLE 1: Difference between the classical PIM and the HAM when $\tau=0.001$ and $\tau=0.005$.

\begin{tabular}{|c|c|c|c|c|}
\hline \multirow{3}{*}{$t$} & \multicolumn{2}{|c|}{$\tau=0.001$} & \multicolumn{2}{|c|}{$\tau=0.005$} \\
\hline & \multicolumn{2}{|c|}{ Maximum of absolute error } & \multicolumn{2}{|c|}{ Maximum of absolute error } \\
\hline & $\Delta=\mid$ Exact - HAM $\mid$ & $\Delta=\mid$ Exact - PIM $\mid$ & $\Delta=\mid$ Exact - HAM $\mid$ & $\Delta=\mid$ Exact - PIM $\mid$ \\
\hline 0.1 & 0.001825 & 0.001862 & 0.001542 & 0.004271 \\
\hline 0.2 & 0.000725 & 0.002339 & 0.001797 & 0.010446 \\
\hline 0.3 & 0.000979 & 0.003806 & 0.002857 & 0.019874 \\
\hline 0.4 & 0.000873 & 0.001949 & 0.003236 & 0.010663 \\
\hline 0.5 & 0.000818 & 0.002270 & 0.001147 & 0.010949 \\
\hline 0.6 & 0.001011 & 0.001955 & 0.001128 & 0.008018 \\
\hline
\end{tabular}

TABLE 2: Difference between the classical PIM and the HAM when $\tau=0.05$ and $\tau=0.1$.

\begin{tabular}{ccc} 
& $\Delta=\mid$ Exact - HAM $\mid$ & $\Delta=\mid$ Exact - PIM $\mid$ \\
\hline 0.1 & 0.006067 & 0.029001 \\
0.2 & 0.015968 & 0.084366 \\
0.3 & 0.036130 & 0.186369 \\
0.4 & 0.031070 & 0.272813 \\
0.5 & 0.007711 & 0.295470 \\
0.6 & 0.008237 & 0.544970
\end{tabular}

$\tau=0.1$

Maximum of absolute error

$\begin{array}{cc}\Delta=\mid \text { Exact }- \text { HAM } \mid & \Delta=\mid \text { Exact }- \text { PIM } \mid \\ 0.011791 & 0.049254 \\ 0.033981 & 0.137331 \\ 0.077718 & 0.283644 \\ 0.086969 & 0.436117 \\ 0.077946 & 0.463685 \\ 0.100736 & 0.710333\end{array}$


As the time step $\tau$ becomes smaller, both the classical PIM and the HAM have higher precision; however, we can see clearly in Figure 3 that the HAM is still better than the classical PIM. Furthermore, from the comparison of (49) and (40), it can be seen that the computation cost of the proposed method is less than the classical PIM.

\section{Conclusions}

In this paper, it is shown that the proposed method can solve the Burgers equation successfully. The numerical comparisons presented demonstrate that the solutions obtained by the classical PIM are not valid for large time step; the method proposed in this paper can obtain higher accuracy than the classical PIM even if the time step is small. Although the method proposed aims at Burgers equation, it is a promising tool for other nonlinear problems.

\section{Acknowledgment}

This work is supported by the National Key Technologies R \& D Program of China under Grant no. 2012BAD35B02 and the National Natural Science Foundation of China under Grant no. 41171337.

\section{References}

[1] S. J. Liao, On the proposed homotopy analysis technique for nonlinear problems and its applications [Ph.D. dissertation], Shanghai Jiao Tong University, 1992.

[2] S. J. Liao, "An approximate solution technique not depending on small parameters: a special example," International Journal of Non-Linear Mechanics, vol. 30, no. 3, pp. 371-380, 1995.

[3] S. J. Liao, "A kind of approximate solution technique which does not depend upon small parameters-II. An application in fluid mechanics," International Journal of Non-Linear Mechanics, vol. 32, no. 5, pp. 815-822, 1997.

[4] S. J. Liao, "Homotopy analysis method: a kind of nonlinear analytical technique not depending on small parameters," Shanghai J. Mech, vol. 18, no. 3, pp. 196-200, 1997.

[5] S. J. Liao, Beyond Perturbation: Introduction to the HomoTopy Analysis Method, Champan and Hall/CRC Press, Boca Raton, Fla, USA, 2003.

[6] M. Sajid and T. Hayat, "The application of homotopy analysis method to thin film flows of a third order fluid," Chaos, Solitons \& Fractals, vol. 38, no. 2, pp. 506-515, 2008.

[7] M. Sajid, T. Hayat, and S. Asghar, "Comparison between the HAM and HPM solutions of thin film flows of non-Newtonian fluids on a moving belt," Nonlinear Dynamics, vol. 50, no. 1-2, pp. 27-35, 2007.

[8] S. J. Liao, "On the homotopy analysis method for nonlinear problems," Applied Mathematics and Computation, vol. 147, no. 2, pp. 499-513, 2004.

[9] S. Abbasbandy, Y. Tan, and S. J. Liao, "Newton-homotopy analysis method for nonlinear equations," Applied Mathematics and Computation, vol. 188, no. 2, pp. 1794-1800, 2007.

[10] T. Hayat, M. Khan, and M. Ayub, "On the explicit analytic solutions of an Oldroyd 6-constant fluid," International Journal of Engineering Science, vol. 42, no. 2, pp. 123-135, 2004.
[11] S. Abbasbandy, "The application of homotopy analysis method to nonlinear equations arising in heat transfer," Physics Letters A, vol. 360, no. 1, pp. 109-113, 2006.

[12] A. H. Nayfeh, Perturbation Methods, John Wiley \& sons, New York, NY, USA, 2000.

[13] J. A. Murdock, Perturbation Methods, Cambridge Texts in Applied Mathematics, Cambridge University Press, Cambridge, UK, 1991.

[14] J.-H. He, "Asymptotic methods for solitary solutions and compactons," Abstract and Applied Analysis, vol. 2012, Article ID 916793, 130 pages, 2012.

[15] J.-H. He, "Addendum: new interpretation of homotopy perturbation method," International Journal of Modern Physics B, vol. 20, no. 18, pp. 2561-2568, 2006.

[16] S.-L. Mei, "Construction of target controllable image segmentation model based on homotopy perturbation technology," Abstract and Applied Analysis, vol. 2013, Article ID 131207, 8 pages, 2013.

[17] W. X. Zhong, "On precise integration method," Journal of Computational and Applied Mathematics, vol. 163, no. 1, pp. 59-78, 2004.

[18] W. X. Zhong, "Precise time-integration method for structural dynamics," Journal of Dalian University of Technology, vol. 34, no. 2, pp. 131-136, 1994.

[19] W. X. Zhong, "Combined method for the solution of asymmetric Riccati differential equations," Computer Methods in Applied Mechanics and Engineering, vol. 191, no. 1-2, pp. 93-102, 2001.

[20] S. Bertoluzza and G. Naldi, "A wavelet collocation method for the numerical solution of partial differential equations," Applied and Computational Harmonic Analysis, vol. 3, no. 1, pp. 1-9, 1996.

[21] S.-L. Mei, Q.-S. Lu, and S.-W. Zhang, "Adaptive wavelet precise integration method for partial differential equations," Chinese Journal of Computational Physics, vol. 21, no. 6, pp. 523-530, 2004.

[22] S.-L. Mei, Q.-S. Lu, S.-W. Zhang, and L. Jin, "Adaptive interval wavelet precise integration method for partial differential equations," Applied Mathematics and Mechanics, vol. 26, no. 3, pp. 364-371, 2005.

[23] S.-L. Mei, H.-L. Lv, and Q. Ma, “Construction of interval wavelet based on restricted variational principle and its application for solving differential equations," Mathematical Problems in Engineering, vol. 2008, Article ID 629253, 14 pages, 2008.

[24] D.-C. Wan and G.-W. Wei, "The study of quasi wavelets based numerical method applied to burgers' equations," Applied Mathematics and Mechanics, vol. 21, no. 10, pp. 1099-1110, 2000.

[25] G. W. Wei, "Quasi wavelets and quasi interpolating wavelets," Chemical Physics Letters, vol. 296, no. 3-4, pp. 253-258, 1998. 


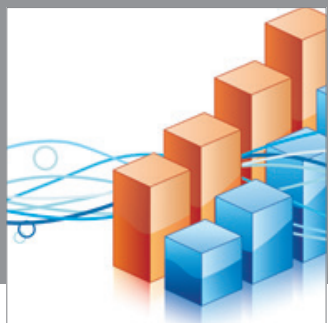

Advances in

Operations Research

mansans

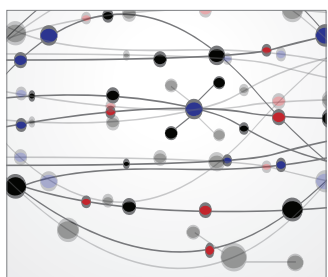

The Scientific World Journal
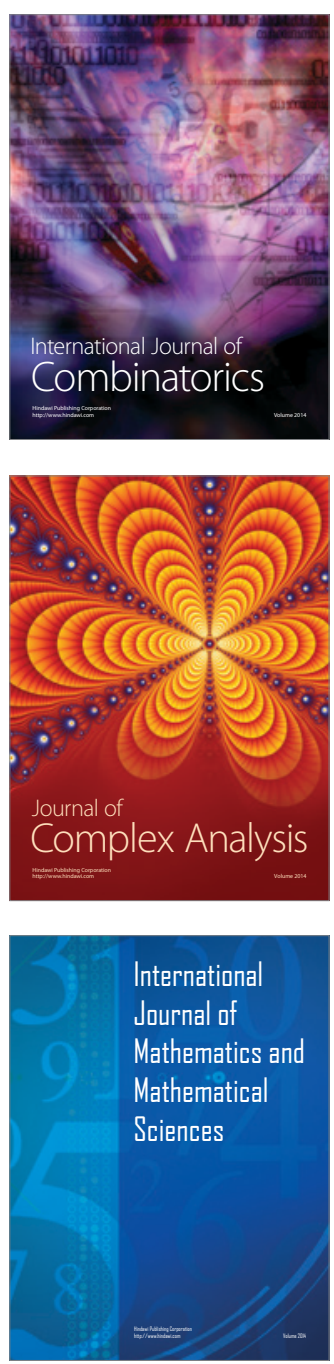
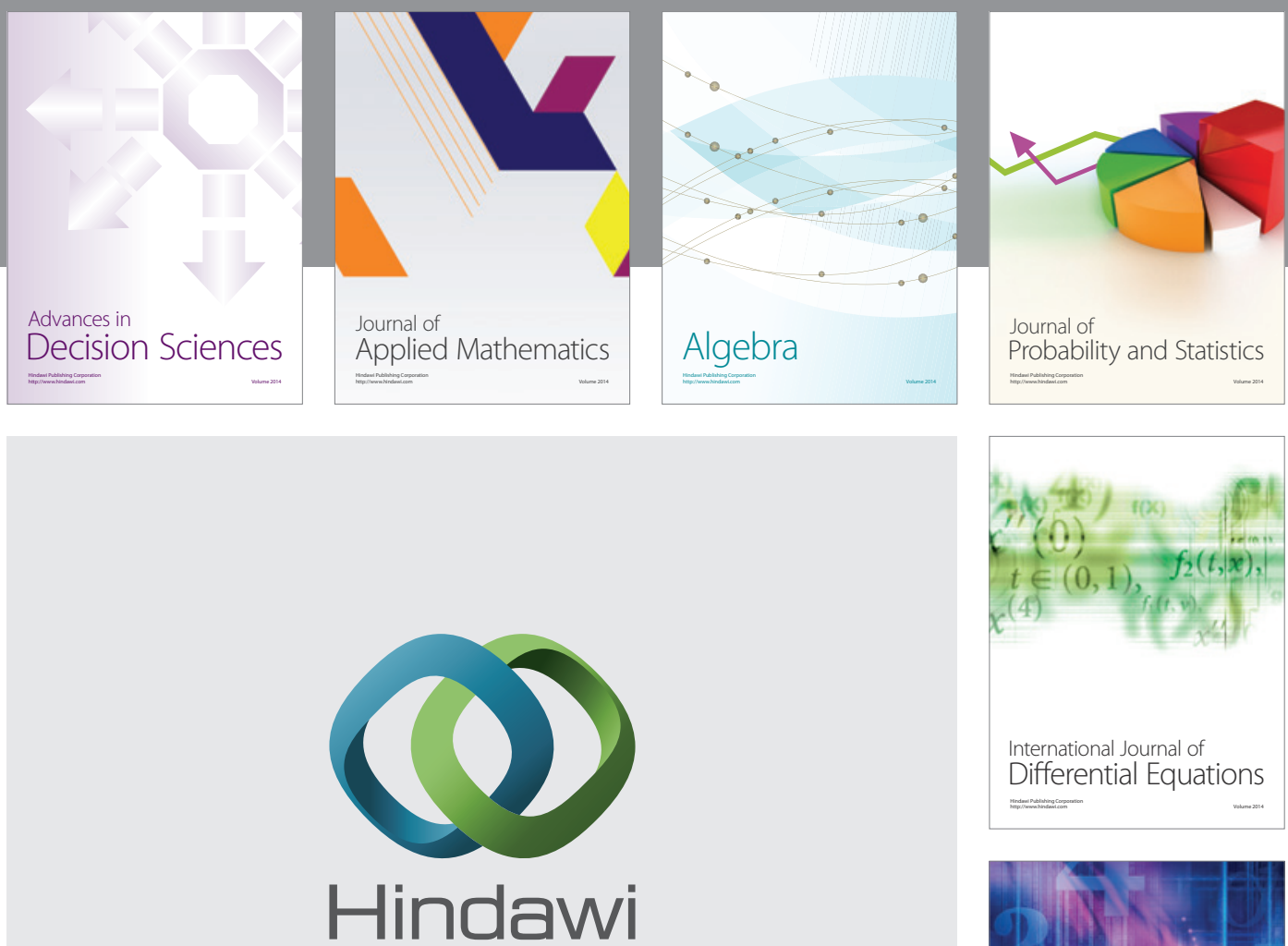

Submit your manuscripts at http://www.hindawi.com
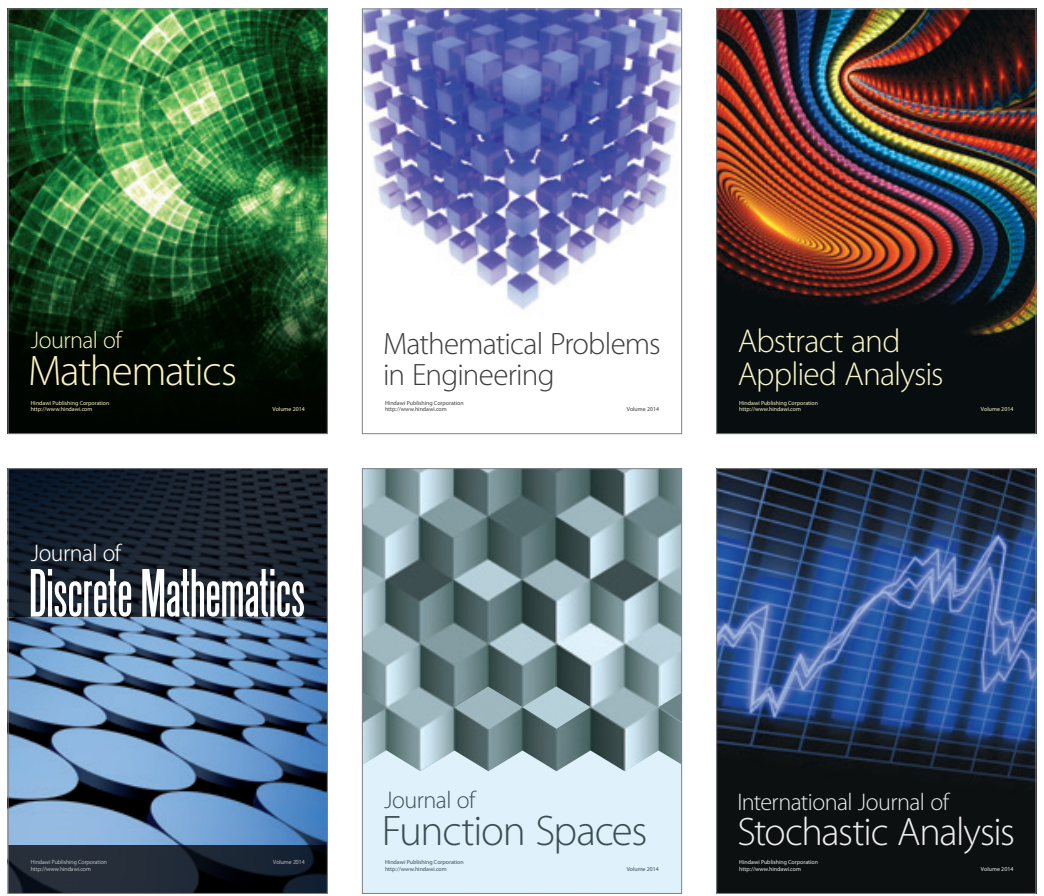

Journal of

Function Spaces

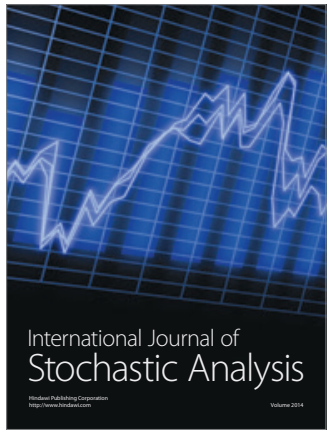

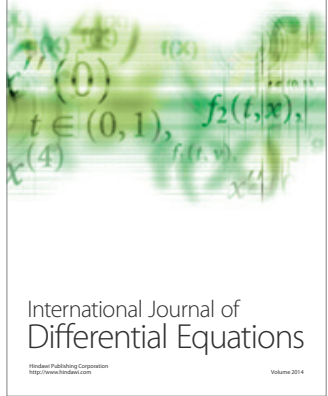
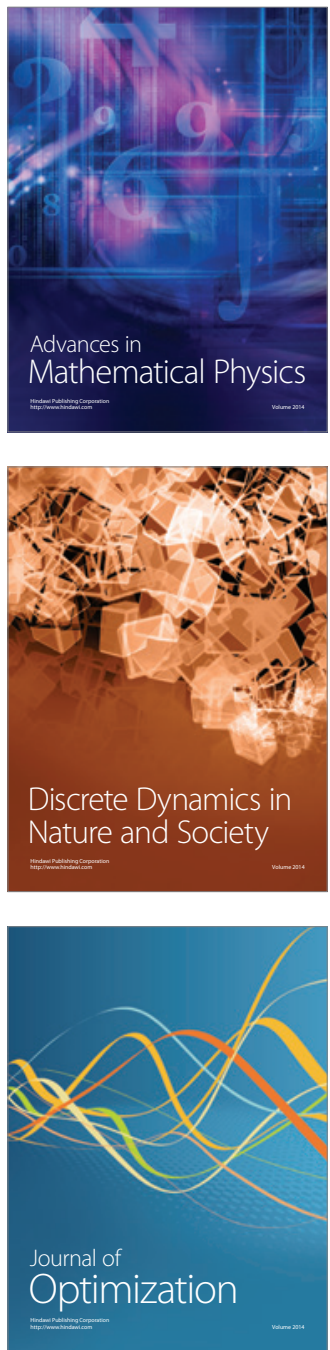